\title{
Missed opportunities for diagnosing primary HIV infection
}

\author{
D Sudarshi, ${ }^{1}$ D Pao, ${ }^{1}$ G Murphy, ${ }^{2}$ J Parry, ${ }^{2}$ G Dean, ${ }^{1}$ M Fisher ${ }^{1}$
}

${ }^{1}$ Department of HIV/GU Medicine, Brighton and Sussex University Hospitals, Brighton, UK: ${ }^{2}$ Virus Reference Department, Centre for Infections, Health Protection Agency

Correspondence to: Dr Darshan Sudarshi, Department of HIV/GU Medicine, Lawson Unit, Eastern Road, Brighton, BN2 5BE, UK; darsh@ doctors.org.uk

MF devised the study. GD created the primary HIV infection (PHI) cohort used in the study. GM and JP were responsible for the laboratory testing for PHI. DS collected the data. DS and DP analysed the data, and DS and MF wrote the manuscript. All authors commented on the final manuscript. $M F$ is the guarantor.

Accepted 22 October 2007 Published Online First 30 October 2007

\begin{abstract}
Objective: To investigate the extent to which primary HIV infection (PHI) presents to healthcare providers and the degree to which it is unrecognised.

Methods: All individuals diagnosed with having recent HIV infection between 2003 and 2005 were identified (based on the following criteria: an evolving antibody response, negative HIV test within 18 months or a serological testing algorithm for recent HIV seroconversion). Symptoms of PHI and previous presentation to other healthcare providers were ascertained from HIV clinic notes and laboratory records (a single laboratory performing all of the HIV tests in the area).
\end{abstract}

Results: Of the 108 subjects, 103 (95\%) were male and $93(86 \%)$ were men who had sex with men. A total of 76 of the 108 individuals $(70 \%)$ reported symptoms of seroconversion. Of these, $40(53 \%)$ presented to a healthcare provider during the symptomatic period. Of these, $21(52 \%)$ were diagnosed with having $\mathrm{PHI}$ at first presentation. In the 19 patients (48\%) in which a diagnosis of having $\mathrm{PHI}$ was not made at first presentation, 15 were seen in primary care, 3 in accident and emergency, and 1 in genitourinary medicine (GUM). Conclusions: The diagnosis of PHI is often missed. Individuals in high-risk groups need to be informed to access healthcare when they experience symptoms of seroconversion. Non-HIV/GUM healthcare providers (especially primary care) may benefit from training in case recognition to improve rates of diagnosis.

The annual number of new diagnoses of HIV continues to rise in the $\mathrm{UK}^{1}$ and elsewhere in the developed world. ${ }^{23}$ Although most new diagnoses are of chronic infections, the number of recently acquired infections in men who have sex with men (MSM) is thought to be either stable $e^{4}$ or increasing. ${ }^{5}$ Of those individuals diagnosed with having chronic infection, a significant proportion are diagnosed "late" and such advanced immunosuppression represents the most common cause of HIV related mortality in the UK. ${ }^{6}$

An estimated $50-90 \%$ of individuals with primary HIV infection (PHI) are thought to develop a symptomatic seroconversion illness. ${ }^{78}$ However, these symptoms are non-specific in nature and may mimic many common febrile illnesses. ${ }^{89}$ Correct diagnosis at this stage of HIV infection is important: it may be the only chance to diagnose HIV in an individual prior to developing advanced disease; it allows consideration of early therapeutic intervention, which may represent a unique window; ${ }^{10}$ and it offers a significant opportunity to prevent onward transmission at a time of increased infectiousness. ${ }^{11} 12$ The extent to which $\mathrm{PHI}$ presents to healthcare providers and the degree to which it is recognised in the UK is unknown. We report a study investigating missed opportunities for diagnosing PHI.

\section{METHODS}

Patients with recent HIV infection (RHI) between 2003 and 2005 were identified from the HIV clinical database. RHI was defined by one or more of the following: a previous negative HIV antibody test within 18 months, an evolving Western blot or antibody response, or by application of a serological testing algorithm for recent HIV seroconversion (STARHS). ${ }^{13}$

At initial presentation to the HIV clinic, the following data were collected: recall of any seroconversion symptoms (for example, fever, rash, sore throat ${ }^{14}{ }^{15}$ ); timing of the seroconversion illness and any self reported illness prior presentation to healthcare providers.

For those individuals who did not self report healthcare presentation, we analysed laboratory records to determine whether any blood tests had been requested by healthcare providers (for example, general practitioner (GP), accident and emergency department (A\&E)) at the time of seroconversion symptoms before presentation to the HIV clinic.

\section{RESULTS}

Of 356 individuals diagnosed with having anti-HIV antibody positive during the study period, 117 $(33 \%)$ were classified as RHI. Clinic notes were accessible for 108 (92\%). Of these, 103 (95\%) were male and 93 (86\%) were MSM. RHI was identified by application of the STARHS assay in 60 (56\%), a previous negative antibody test within 18 months in $11(10 \%)$, an evolving serological response in 3 $(3 \%)$ or by combination of the above in 34 (31\%).

Altogether, 76 of the 108 patients (70\%) reported symptoms of seroconversion, of whom $91 \%$ were MSM. The most common symptoms reported were fever $(55 \%)$, rash $(37 \%)$, sore throat $(33 \%)$, diarrhoea (28\%), swollen glands (21\%), arthralgias $(12 \%)$, headache $(11 \%)$ and flu-like symptoms $(46 \%)$. Of the 76 symptomatic individuals, 40 $(53 \%)$ accessed health care at the time. Of these, 21 $(52 \%)$ patients were correctly diagnosed with having PHI at first presentation: $12(57 \%)$ by genitourinary medicine (GUM); 5 (24\%) by other hospital teams (4 by general medical teams); and 4 (19\%) by their GP.

Altogether, 19 of 40 patients (48\%) had a prior presentation to health care at the time of seroconversion, yet a diagnosis of PHI was missed (details in table 1); 17 (89\%) of these were MSM. Fifteen $(79 \%)$ had presented to their GP, three 
$(16 \%)$ to A\&E and one (5\%) to GUM. Of the 19 "missed" cases, 13 were identified as missed because the patient reported prior presentation to health care with symptoms suggestive of seroconversion. Diagnoses the patients recall being given at these consultations included bacterial infection $(n=4)$, tonsillitis $(n=2)$, glandular fever $(n=1)$, viral infection $(n=1)$, thrombocytopenia of unknown cause $(n=1)$ and in four cases no diagnosis was given. Six of the 19 missed cases were identified as such because laboratory records showed blood tests requested by healthcare providers at the time of the reported seroconversion symptoms. In one of these six cases a HIV test had been performed. This patient had presented to GUM where a HIV test (a fourth generation combined antibody/antigen test) was negative at the time of presentation but the patient was not retested until a subsequent presentation 3 months later.

\section{DISCUSSION}

This study shows that the diagnosis of PHI is often missed, and highlights two points at which this occurs. First, almost half $(47 \%)$ of the symptomatic individuals did not seek medical attention when they were experiencing classical seroconversion symptoms. Second, in almost half (48\%) of the symptomatic individuals that presented to health care the diagnosis of PHI was not made; the majority of these missed diagnoses were in primary care (79\%). Despite the fact that MSM represents the major risk category for acquiring HIV infection in the UK, ${ }^{1}$ our results show that PHI remains largely undiagnosed in this group.

\section{Comparison with previous studies}

This is the first UK study of its kind. Melzer et al previously showed that PHI may not always be diagnosed by healthcare providers: they anonymously tested the sera of 238 attendees with "symptoms consistent with seroconversion" and found two cases of undiagnosed PHI. ${ }^{16}$ Our findings are also consistent with two North American studies that showed that PHI is infrequently considered as the diagnosis when symptomatic patients first present to health care. ${ }^{89}$

The other principal finding in our study was that only $53 \%$ of the symptomatic patients accessed health care at the time of their seroconversion illness. This is in contrast to Schacker et al, ${ }^{8}$ who showed in a cohort of 46 patients with PHI (of whom the majority were MSM) that $94 \%$ sought health care when they had symptoms. Of note, that study was completed before the advent of highly active antiretroviral treatment and in an entirely different healthcare system. Furthermore, our findings are supported by a recent questionnaire study in North America, which showed that although most HIV negative men could identify symptoms of PHI relatively few would access health care for such symptoms. ${ }^{17}$

Table 1 Details of the 19 missed opportunities that presented to healthcare

\begin{tabular}{|c|c|c|c|c|}
\hline Case & Ethnicity, sexuality, gender & Symptoms & $\begin{array}{l}\text { Diagnosis patient recalls } \\
\text { being given }\end{array}$ & $\begin{array}{l}\text { Healthcare } \\
\text { provider }\end{array}$ \\
\hline 1 & White MSM & $\begin{array}{l}\text { Fever, sore throat, swollen glands, } \\
\text { arthralgia }\end{array}$ & Tonsillitis & GP \\
\hline 2 & White heterosexual female & \multicolumn{2}{|c|}{ Fever, sore throat, diarrhoea, headacheTonsillitis } & A\&E \\
\hline 3 & White heterosexual female & $\begin{array}{l}\text { Rash, sore throat, swollen glands, } \\
\text { weight loss }\end{array}$ & Glandular fever & $\mathrm{GP}$ \\
\hline 4 & White MSM & Fever, rash, flu-like symptoms & Viral infection & GP \\
\hline 5 & White MSM & $\begin{array}{l}\text { Fever, abdominal pain, flu-like } \\
\text { symptoms }\end{array}$ & Low platelets & GP \\
\hline 6 & White MSM & $\begin{array}{l}\text { Fever, sore throat, malaise, weight } \\
\text { loss, headaches }\end{array}$ & Bacterial infection & $\mathrm{GP}$ \\
\hline 7 & White MSM & Fever, sore throat & Bacterial infection & GP \\
\hline 8 & White MSM & Fever, sore throat, flu-like symptoms & Bacterial infection & GP \\
\hline 9 & White MSM & Rash, sore throat, flu-like symptoms & Bacterial infection & GP \\
\hline 10 & White MSM & Fever, rash, flu-like symptoms & Not specified & GP \\
\hline 11 & White MSM & $\begin{array}{l}\text { Fever, rash, sore throat, } \\
\text { lymphadenopathy, mouth sores }\end{array}$ & Not specified & $\mathrm{GP}$ \\
\hline 12 & White MSM & Flu-like symptoms & Not specified & GP \\
\hline 13 & White MSM & Fever, sore throat, diarrhoea, & Not specified & A\&E \\
\hline \multicolumn{5}{|c|}{ Cases 14-19: laboratory evidence of prior presentation to health care with seroconversion symptoms } \\
\hline Case & Ethnicity, sexuality, gender & Clinical information on blood form & $\begin{array}{l}\text { Laboratory tests } \\
\text { requested }\end{array}$ & $\begin{array}{l}\text { Healthcare } \\
\text { provider }\end{array}$ \\
\hline 14 & White MSM & Rash, streptococcal & ASOT titre & GP \\
\hline 15 & White MSM & $\begin{array}{l}\text { History malaise, cough, swollen } \\
\text { glands }\end{array}$ & $\begin{array}{l}\text { FBC, U\&E, LFTs, CMV+ EBV } \\
\text { serology, monospot }\end{array}$ & $\mathrm{GP}$ \\
\hline 16 & White MSM & Fever returning from Kenya & FBC, U\&E, LFTs, blood film & GP \\
\hline 17 & White MSM & Diarrhoea, malaise & $\begin{array}{l}\text { FBC, U\&E, LFTs, ESR, stool } \\
\text { culture }\end{array}$ & $\mathrm{GP}$ \\
\hline \multirow[t]{2}{*}{18} & White bisexual male & Sore throat 3 weeks & $\begin{array}{l}\text { FBC, U\&E, LFTs, monospot } \\
\text { test }\end{array}$ & A\&E \\
\hline & & Infectious mononucleosis & Throat culture & \\
\hline 19 & White MSM & HIV & Fourth generation HIV test & GUM clinic \\
\hline
\end{tabular}

A\&E, accident and emergency; ASOT, antistreptolysin-0-test; CMV, cytomegalovirus; EBV, Epstein-Barr virus; ESR, erythrocyte sedimentation rate; FBC, full blood count; GP, general practitioner; GUM, genitourinary medicine; LFT, liver function tests; MSM, men who have sex with men; U\&E, urea and electrocytes 


\section{Limitations}

Our study used patient's recall of prior presentation to health care and therefore reporting may not be completely accurate. The effect of this reporting bias is highlighted by identification of further missed opportunities using laboratory records (table 1, cases 14-19). In this study, a significant proportion of individuals did not access health care through the duration of their PHI. This includes 32 individuals who did not report seroconversion symptoms, as well as 36 symptomatic individuals who did not access health care at the time. We were unable to comment on the specific reasons why these individuals did not access health care initially or the factors leading them eventually to present. In addition, further research is needed to explore strategies to detect those individuals who remain undiagnosed in the community. From a provider perspective, it was not possible to access primary healthcare records because of patient confidentiality. Hence we could not clarify if healthcare professionals had considered HIV as a diagnosis, completed a risk assessment (including knowledge of sexual orientation) or if HIV testing was offered and refused at time of presentation. Of relevance, a survey of over 13000 MSM showed that only $28 \%$ believed that their GPs had knowledge of their sexuality. ${ }^{18}$ Our study was conducted in an urban centre with a large white MSM community and, therefore, may not be representative of those populations faced by many primary care physicians. However, our findings show that in two instances the diagnosis of PHI was missed in heterosexual females, which suggests high degree vigilance is needed for diagnosis of PHI in all populations.

\section{Implications}

Strategies to improve these outcomes need to consider both patient and provider. From a patient perspective, the likelihood of future diagnosis could be improved by encouraging at-risk groups (for example, MSM) to access health care when they experience symptoms of seroconversion or following high risk exposure. From a provider perspective, training for non-HIV/ GUM specialists should include recognition of the acute seroconversion illness. In the current era, reducing stigma and enabling discussion of potential risk factors for HIV acquisition is likely to facilitate the diagnosis of PHI. In this study, a combination of awareness of sexuality with recognition of classical symptoms of PHI may have enabled the diagnosis in $16 / 19(84 \%)$ of missed cases that had presented to health care.

\section{Key messages}

- Detection of primary HIV infection (PHI) has important benefits to individual and public health.

- The opportunity to diagnose PHI is often missed, despite the frequent occurrence of a seroconversion illness.

- Either patients do not access health care when they have typical seroconversion symptoms or healthcare providers (notably primary care) do not make the diagnosis when patients present to them with suggestive symptoms.
In one missed case (case 19, table 1), the diagnosis of PHI was not made even though a HIV test had been performed. Although the latest fourth generation combined antibody/ antigen test provides improved sensitivity for detecting PHI, those presenting very early after onset of symptoms may still be missed. In such instances, where PHI is strongly suspected, follow up HIV testing 1-2 weeks later is recommended.

As HIV testing becomes increasingly "normalised", ${ }^{19}$ barriers to performing HIV tests in non-GUM settings will hopefully be overcome. Given that individuals with PHI are more infectious and contribute disproportionately to onward transmission, ${ }^{11}{ }^{12} \mathrm{a}$ significant opportunity exists to improve both individual and public health.

Acknowledgements: We gratefully acknowledge the contributions of Gary Homer and Dennis Mcelborough (Virology, Royal Sussex County Hospital, UK).

Competing interests: None.

\section{REFERENCES}

1. Health Protection Agency Data. A complex picture - HIV and other sexually transmitted infections in the United Kingdom: 2006. http://www.hpa.org.uk/hpa/ publications/hiv sti 2006/pdf/Mtl FC report.pdf (accessed 22 Aug 2007).

2. National Centre in HIV Epidemiology and Clinical Research. HIV/AIDS, viral hepatitis and sexually transmissible infections in Australia, annual surveillance report, 2004. http://web.med.unsw.edu.au/nchecr/Downloads/04ansurvrpt_2.pdf laccessed 22 Aug 2007).

3. Centres for Disease Control and Prevention. Increases in HIV diagnoses - 29 States, 1999-2002. MMWR 2003;52:1145-8.

4. Murphy G, Charlett A, Jordan LF, et al. HIV incidence appears constant in men who have sex with men despite widespread use of effective antiretroviral therapy. AIDS 2004;18:265-72.

5. Dukers NTM, Spaargaren J, Geskus RB, et al. HIV incidence on the increase among homosexual men attending an Amsterdam sexually transmitted disease clinic: using a novel approach for detecting recent infections. AIDS 2002;16:F19-24.

6. BHIVA (British HIV Association) audits and standards. Preliminary findings of the mortality audit, 2006. http://www.bhiva-clinical audit.org.uk/Portals/_Rainbow/ Documents/06CVDprelimMortal.ppt (accessed 10 May 2006).

7. Tindall B, Barker S, Donovan B, et al. Characterisation of the acute clinical illness associated with human immunodeficiency virus infection. Arch Intern Med 1988;148:945-9.

8. Schacker T, Collier AC, Hughes J, et al. Clinical and epidemiologic features of primary HIV infection. Ann Intern Med 1996;125:257-64.

9. Weintrob AC, Giner J, Menezes P, et al. Infrequent diagnosis of primary human immunodeficiency virus infection: missed opportunities in acute care settings. Ant Intern Med 2003;163:2097-100.

10. Rosenberg ES, Altfeld M, Poon SH, et al. Immune control of HIV-1 after early treatment of acute infection. Nature 2000;407:523-6.

11. Wawer MJ, Gray RH, Sewankambo NK, et al. Rates of HIV-1 transmission per coital act, by stage of HIV-1 infection, in Rakai, Uganda. JID 2005:1403-9.

12. Pilcher CD, Tien HC, Eron JJ Jr, et al. Brief but efficient: acute HIV infection and the sexual transmission of HIV. JID 2004;189:1785-92.

13. Janssen RS, Satten GA, Stramer SL, et al. New testing strategy to detect early HIV1 infection for use in incidence estimates and for clinical and prevention purposes. JAMA 1998;280:42-8.

14. Hecht F, Busch MP, Rawal B, et al. Use of laboratory tests and clinical symptoms for identification of primary HIV infection. AIDS 2002;16:1119-29.

15. Daar ES, Little S, Pitt J, et al. Diagnosis of primary HIV-1 infection. Ann Intern Med 2001;134:25-9.

16. Melzer M, Brown M, Mullen J, et al. Undiagnosed symptomatic primary HIV infections in South London. J Infect 2001:42:297-8.

17. Stekler J, Collier AC, Holmes KK, et al. Primary HIV infection education: knowledge and attitudes of HIV-negative men who have sex with men attending a public health sexually transmitted disease clinic. JAIDS 2006;42:123-6.

18. Sigma research report. Doctoring gay men: exploring the contributions of general practice. 2004. http://www.sigmaresearch.org.uk/downloads/report04d.pdf (accessed 12 Sep 2006).

19. Bayer R, Fairchild AL. Changing the paradigm for HIV testing-the end of exceptionalism. N Engl J Med 2006;355:647-9. 